\title{
Productividad y crecimiento del sector del Transporte en España. Análisis por subsectores.
}

\author{
Javier Anibarro García \\ Experto Senior del Área de Economía y Política del Transporte - INECO \\ Fernando Cámara de la Peña \\ Universidad Carlos III de Madrid e INECO
}

\section{RESUMEN}

La crisis económica ha puesto de manifiesto la fragilidad de un modelo de crecimiento basado en el uso intensivo de factores con baja productividad. La ponencia presenta este fenómeno en el sector del "Transporte y Almacenamiento" español, analiza sus causas y sugiere cursos de actuación.

Pese a ese a ser un país intensivo en movilidad (toneladas-km/PIB y viajeros-km/PIB), con un elevado número de vehículos y empresas, el sector español representa sólo un $40 \%$ del valor medio de la producción y un $60 \%$ del empleo del de las cuatro grandes economías europeas (G4: Alemania, Francia, Italia y el Reino Unido). Por tanto, el sector presenta bajos niveles de productividad. La convergencia del sector español a los parámetros del sector en el G4 permitiría aumentar la producción. Una parte de este crecimiento provendría de aumentar la productividad, pero no todo. ¿Cómo conseguirlo?

Para dar respuesta a esta pregunta, el estudio disecciona las productividades del sector español: por subsectores que lo integran y por tamaño de las empresas (número de ocupados) y las compara con las del resto de países. El análisis extrae que la mayor parte del desfase en productividad del sector español se produce en el "Transporte Terrestre y por Tubería" mientras el "Almacenamiento y Actividades Anexas" aunque tiene un volumen de la producción relativamente reducido, presenta productividades similares e incluso superiores a la de los países del G4. La menor productividad del Transporte Terrestre se explica por su estructura interna, con una desproporcionada presencia de microempresas de baja productividad que afecta al volumen de producción total. En el Almacenamiento y Actividades Anexas, la baja productividad no explica la baja producción. Por lo que, aunque en ambos casos la producción es baja, son realidades diferentes que deben ser tratadas de forma diferente.

Cabe concluir que la vía para incrementar el valor de la producción en Transporte Terrestre es incrementar la productividad aumentando la dimensión de las empresas, especialmente en transporte de mercancías. El Almacenamiento y Actividades Anexas, por el contrario, no tiene un problema de productividad sino de baja demanda, por lo que su crecimiento pasaría más por el aprovechamiento de las oportunidades de una logística avanzada por parte del tejido industrial y productivo español. 


\section{MOTIVACIÓN Y ANTECEDENTES}

La intensa crisis económica española ha puesto de manifiesto la fragilidad de un modelo de crecimiento basado en la utilización intensiva de recursos de baja productividad, especialmente concentrados aunque no exclusivamente, en la actividad de la construcción por el lado de la oferta, y un crecimiento de la demanda sustentado en un fuerte endeudamiento externo de los agentes privados.

La corrección de los desequilibrios que implica este modelo de crecimiento pasa fundamentalmente por mejorar el saldo exterior de España aumentando las exportaciones y la inversión exterior y por un aumento de la productividad de las actividades económicas. Estos ajustes comenzaron a producirse en España, si bien de forma dramática a partir del año 2008.

En este contexto es especialmente relevante analizar el sector del transporte por varios motivos. En primer lugar porque es, en sí mismo, un sector cuantitativamente importante cuyo Valor Añadido Bruto (VAB) representa algo más del 5\% del PIB español y su empleo una magnitud ligeramente inferior.

Pero más allá de su importancia cuantitativa directa, el transporte y la logística deben desempeñar un papel fundamental en la corrección del modelo de crecimiento español hacia parámetros de crecimiento económico más sanos y sostenibles por su especial importancia para el comercio exterior y por su valor transversal como input que incide en una amplia gama de actividades productivas esenciales desde el sector industrial hasta el turismo.

Esta ponencia se centra en el análisis del sector del "Transporte y Almacenamiento" español desde el punto de vista de su sostenibilidad económica, analizando arrojando luz sobre cuánto, dónde y cómo puede el sector aumentar su productividad y contribuir al reequilibrio económico global del país.

Tradicionalmente los análisis de estructura y coyuntura del sector se han centrado más en las mediciones de movilidad como indicador de su comportamiento. Esta forma de enfocar el análisis del transporte aunque útil para el seguimiento coyuntural y de corto plazo, puede llevar a confusión y errores de política en el medio y largo plazos, especialmente en el nuevo escenario que se presenta para la actividad marcado por la digitalización y la gestión de grandes volúmenes de información.

Por ello esta ponencia analiza el sector de forma diferente, prestando más atención a la estructura del sector, consciente de que la generación de valor es la clave y que este tendrá en el futuro menos relación directa con los volúmenes de tráfico producidos. 


\section{ESTRUCTURA ACTUAL DEL TRANSPORTE EN ESPAÑA}

España es un país intensivo en transporte, Como se puede observar en la figura 1, que refleja los datos de movilidad interior y exterior de los modos terrestres en varios países europeos, el transporte de viajeros tiene una intensidad relativa al PIB similar a la media de la UE, pero en transporte de mercancías esta intensidad se sitúa en niveles muy superiores, siendo esta diferencia incluso mayor con los países europeos más desarrollados.

Fig. 1 - Intensidad del transporte de viajeros y mercancías interior y exterior en modos terrestres (viajeros-km y toneladas-km/1000 Euros constantes) en relación al PIB en España y principales economías de la $U E$.

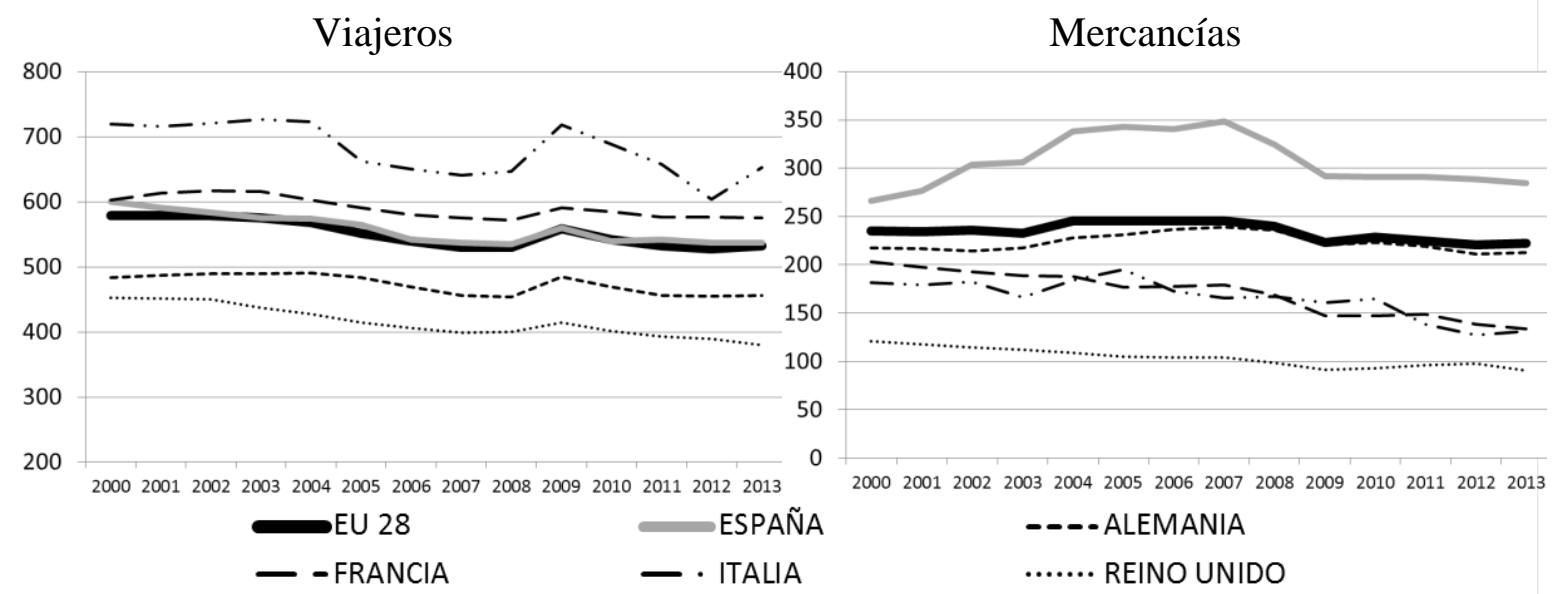

Fuente: Elaboración propia con datos de Eurostat y Anual Macro-Economic Database of the European Comission (AMECO)

En consonancia con lo anterior, la flota de vehículos de transporte de mercancías por carretera es en España relativamente muy superior a la de los países del G4 (Alemania Italia y Reino Unido), mientras que la de autobuses entra dentro de la normalidad, como muestra la siguiente tabla.

Tabla 1 - Stock de vehículos registrados (miles) en España y principales economías de la UE. 2013

\begin{tabular}{lccccc}
\hline & España & Alemania & Francia & Italia & $\begin{array}{c}\text { Reino } \\
\text { Unido }\end{array}$ \\
\hline $\begin{array}{l}\text { Autobuses } \\
\begin{array}{l}\text { Vehículos de } \\
\text { mercancías }\end{array}\end{array}$ & 59,9 & 76,8 & 95,2 & 98,6 & 111,6 \\
\hline
\end{tabular}

Fuente: EU Transport in figures. Statistical pocket book 2015 - Comisión Europea

La figura 2 muestra las principales variables de la estructura económica del sector Transporte y Almacenamiento ${ }^{1}$ en España.

1 Se considera Transporte y Almacenamiento a la sección H de la Nueva Clasificación Nacional de Actividades Económicas (CNAE-2009) 
Destaca, en primer lugar, el contraste entre las elevadas magnitudes de movilidad y flota en España comentadas anteriormente con el bajo nivel de producción o valor añadido del sector que no alcanza ni la mitad del existente en la media de los países del G4. En segundo lugar, también es reseñable que la ocupación (empleo) del sector español es inferior a la media de la de aquellos países. Aunque la diferencia aquí es menor que la que había en términos de producción: un $60 \%$. Un primer corolario de la comparación de estas cifras es que la productividad media (valor de la producción por persona empleada) del sector en España está por detrás de la de los países de referencia. Finalmente, destaca también el elevadísimo número de empresas que operan en España que duplican la media de los países del G-4, lo que implica que las empresas españolas tienen de media menor dimensión que las europeas en lo que a producción y ocupación se refiere.

Fig. 2 - Número de empresas, producción (euros corrientes) y personas empleadas en “Transporte y Almacenamiento” en España y principales economías UE 2013

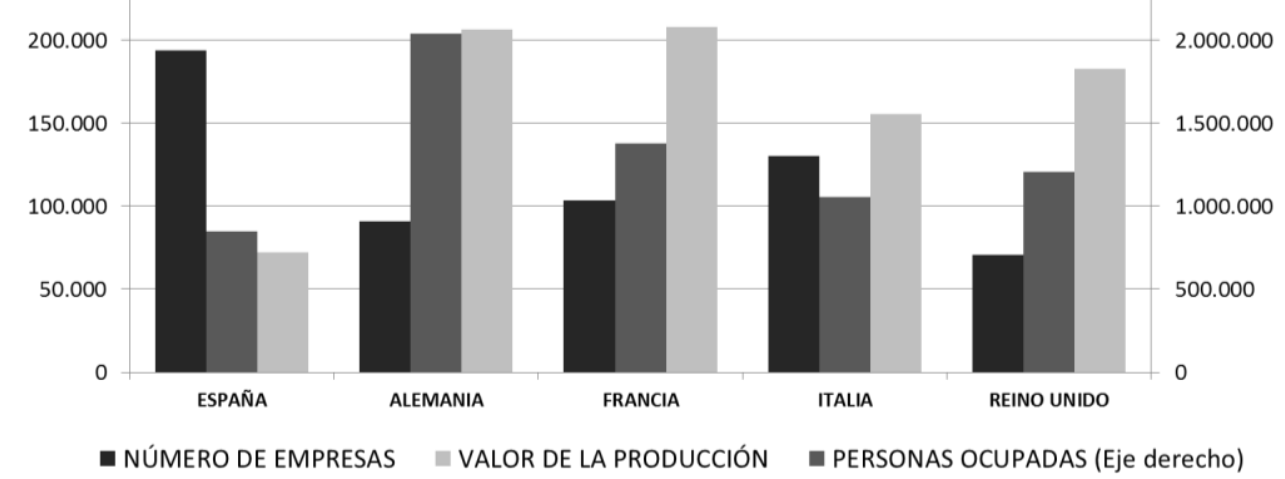

Fuente: Elaboración propia con datos de Eurostat

Para hacerse una idea de la desproporción, la figura 3 muestra cómo sería el sector español si tuviese la misma estructura que el G4 con la población y renta actuales, y con la población actual y un PIB per cápita como el de los países de referencia.

Fig. 3 - Comparación entre magnitudes actuales del sector español y las que le correspondería con la estructura del G-4 (ajustado por población y PIB per cápita). 2013

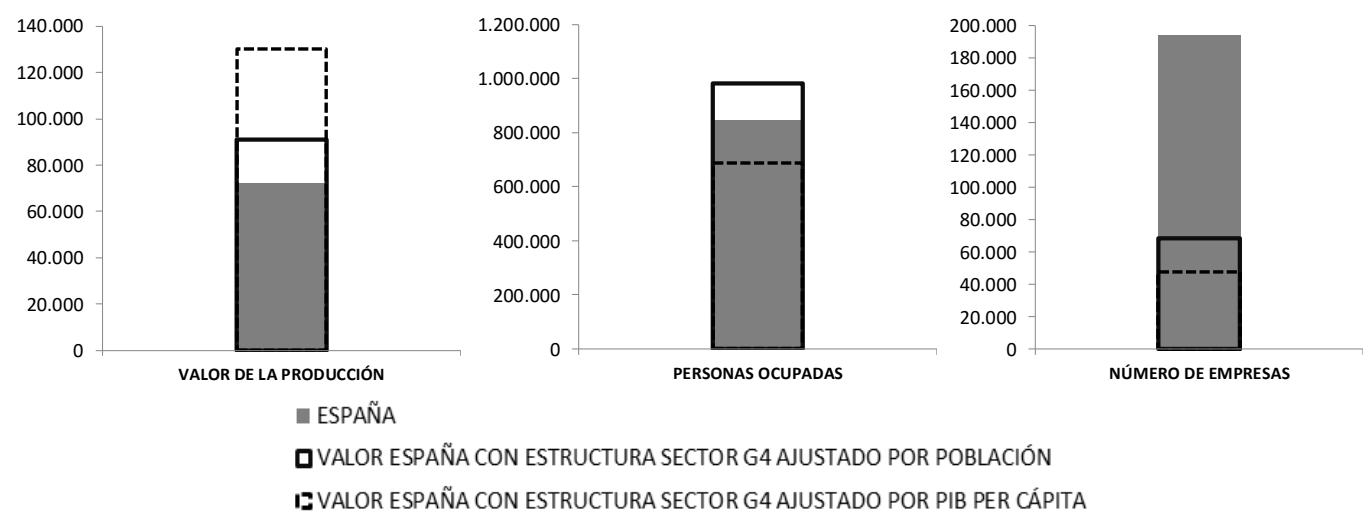

Fuente: Elaboración propia con datos de Eurostat

http://www.ine.es/daco/daco42/clasificaciones/cnae09/cnae_2009_rd.pdf 


\section{POTENCIAL DE CRECIMIENTO DEL SECTOR ESPAÑOL}

De lo analizado se deducen cuatro características especiales del Transporte español:

- $\quad$ ALTA MOVILIDAD: España es un país con alta movilidad y gran número de vehículos comerciales, especialmente de transporte de mercancías,

- $\quad$ BAJA PRODUCCIÓN: Pese a ello, el valor de la producción del sector "Transporte y Almacenamiento" es inusualmente bajo.

- BAJA PRODUCTIVIDAD: El empleo es también menor, pero no tanto, lo que implica menor productividad que los países del G4.

- EMPRESAS PEQUEÑAS: El número de empresas es muy alto, lo que implica menor dimensión media de las empresas.

Esta estructura del sector refleja debilidades relacionadas con las características generales de la economía española citadas en la introducción. Pero tiene también una lectura positiva. Como se ha mostrado en la figura 3, el sector tiene un alto potencial de crecimiento si se producen reformas que lo hagan converger con la estructura del G4. En términos de PIB per cápita, el valor de la producción del sector español debería ser casi un $80 \%$ superior al actual, el número de empleados un $16 \%$ (pues se convergiría también en productividad) y el número de empresas sería un $65 \%$ inferior (-125.000).

El resto de esta ponencia se orienta a responder las siguientes preguntas:

1. ¿Es razonable plantearse una convergencia de la estructura del sector español a la existente en las economías del G4?, y en segundo lugar, si es así,

2. ¿Cómo se debería producir esta convergencia?

Es evidente que existen factores más allá de la estructura y el PIB per cápita que alejan el sector español del de los países del G4. España ocupa una posición más periférica que aquellos y tiene una menor densidad de población. Estos hechos ocasionan una mayor intensidad de la movilidad. También puede citarse la especial importancia del turismo en la economía española, aunque este hecho incide más en el transporte aéreo.

Por otra parte, una parte de las empresas de otros sectores realizan internamente su actividad de transporte. Este transporte no externalizado se registra en los datos de movilidad y flotas, pero no computa como actividad del "Transporte y Almacenamiento", aunque este hecho también puede interpretarse parcialmente como una debilidad del sector para atender competitivamente las necesidades logísticas de esas empresas.

En definitiva, estos factores matizan, pero no anulan a juicio de los autores, la validez de la estructura del sector en las cuatro grandes economías desarrolladas de Europa como referencia para el desarrollo del sector, por lo que cabe preguntarse las causas de este desfase y los caminos de convergencia. 
Para ello se plantea desagregar la estructura del sector en sus componentes principales y analizar cómo se distribuye la actividad por tamaños de las empresas, aproximados éstos por los diferentes segmentos de personas ocupadas en la empresa.

El análisis se centra en los dos principales subsectores que integran el "Transporte y Almacenamiento" que son el "Transporte Terrestre y por Tubería" (principalmente transporte de mercancías y viajeros por carretera, además de ferrocarril, tuberías y otros) y "Almacenamiento y Actividades Anexas" que incorpora la actividad no directamente relacionada con el desplazamiento de personas y mercancías, En general este subsector está más vinculado con la actividad logística y la gestión. Se descarta el análisis del transporte aéreo y marítimo, además de por razones de espacio e importancia (AENA y los puertos forman parte del "Almacenamiento y Actividades Anexas"), por el hecho de que se trata de actividades muy internacionalizadas, donde el mercado y las condiciones de competencia son globales y la relevancia de las condiciones nacionales menor.

La figura 4 muestra el valor de la producción, número de empresas y empleo en estos dos subsectores.

Fig. 4 - Número, valor de la producción (euros corrientes) y personas empleadas en las empresas de "Transporte Terrestre" y "Almacenamiento y Actividades Anexas" en España y principales economías de la UE. 2013

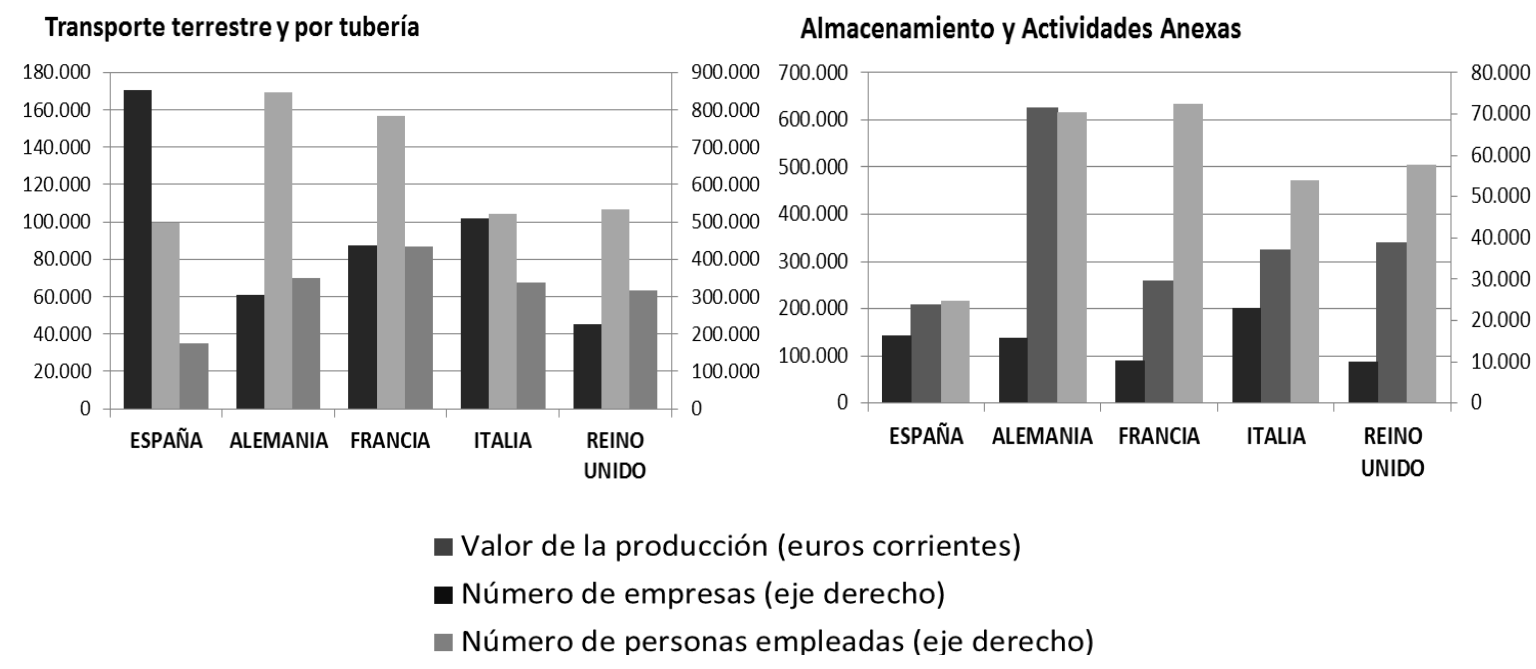

Fuente: Elaboración propia con datos de Eurostat

En ambos subsectores, el valor de la producción es bajo en relación a los países de referencia, como el sector en general. Sin embargo, la estructura de ambos subsectores difiere notablemente.

El sector de Transporte Terrestre y por Tubería tiene un elevadísimo número de empresas, un valor de la producción muy bajo y un número de empleados que no es tan inferior, por lo que la productividad por empleado en este subsector es claramente inferior. Por el contrario, 
el Almacenamiento y Actividades Anexas español tiene un número de empresa en línea con el resto de países, pero, pese a mostrar un valor de la producción inferior, es el que presenta mayor productividad por empleado de entre los cinco países considerados.

La figura 5 ayuda a entender las causas de este diferente comportamiento. En este gráfico se muestra la relación creciente que existe entre tamaño de la empresa (aproximado por el número de empleados) y la productividad por empleado para España y los países del G-4 en ambos subsectores. A medida que crecen las empresas, la productividad crece en ambos subsectores y en ambas referencias geográficas. En general la productividad del Almacenamiento y Actividades Anexas supera al Transporte Terrestre. Pero lo más importante es que la pendiente de la curva española es más pronunciada que la de los demás países. Para los tamaños menores de empresas, las españolas son menos productivas que sus correspondientes europeas. Mientras que las más grandes son incluso más productivas en España que en los demás países de media.

El problema radica en la diferente distribución de las empresas. Cerca del $40 \%$ de la producción en transporte terrestre se concentra empresas con menos de 9 empleados (el tramo de baja productividad) frente al $15 \%$ del G4 mientras que las de mayor tamaño (y productividad) solo concentran el $28 \%$ de la producción frente al $49 \%$ de las europeas. En el almacenamiento y actividades anexas, las empresas de menos de 9 empleados producen el $9 \%$ de la producción total tanto en España como en el G4, mientras que las mayores acumulan el $50 \%$ de la producción en España y el $58 \%$ en el G4. Una estructura más equiparable.

Fig. 5 - Productividad media de las empresas por segmento de ocupación. España y G4 (Alemania, Francia, Italia y Reino Unido). Año 2013

Transporte terrestre y por tubería

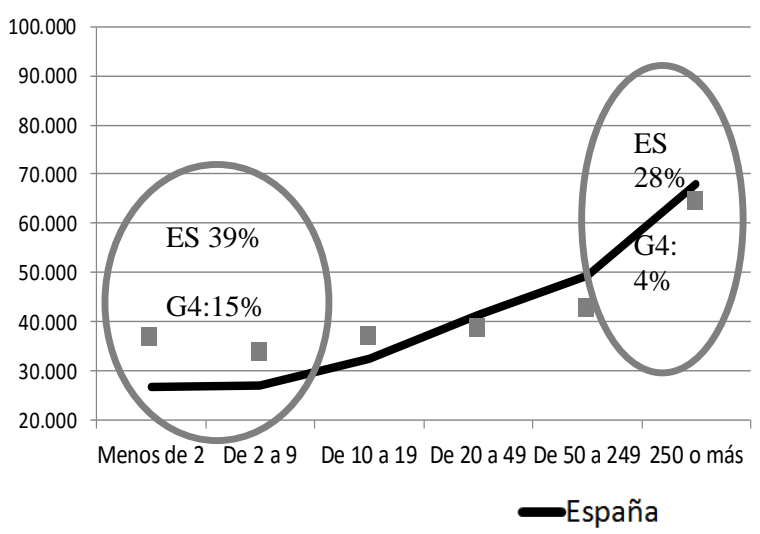

Almacenamiento y actividades anexas

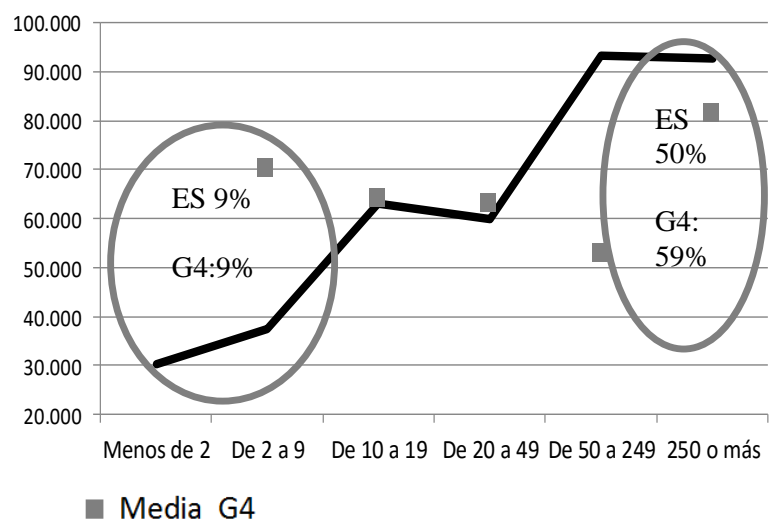

Fuente : Elaboración propia con datos de Eurostat

Un análisis ulterior similar, que no se muestra por cuestión de espacio, muestra cómo el problema del Transporte Terrestre se concentra en el transporte de mercancías por carretera. 


\section{CONCLUSIONES. E IMPLICACIONES DE POLÍTICA}

España es un país con movilidad alta. El volumen de los tráficos es alto en relación a su PIB cuando se compara con otros países europeos similares o de mayor nivel de desarrollo. Una parte de la causas de este hecho puede tener su origen en factores asociados a su geografía como una baja densidad de población y actividad o una posición relativamente periférica respecto a los grandes centros de producción y consumo europeos, entre otros.

Sin embargo lo que llama la atención es que pese a su alta movilidad, el sector del "Transporte y Almacenamiento" que recoge la actividad de las empresas que se dedican principalmente al desplazamiento de personas y mercancías y otras actividades logísticas es inusualmente bajo en comparación con los países que tienen una menor intensidad de transporte. En la ponencia el análisis se centra en las cuatro grandes economías europeas: Alemania, Francia, Italia y el Reino Unido.

Tras diseccionar el sector en sus principales subsectores y características (número de empresas, ocupados y productividad), se extrae la conclusión de que las pequeñas empresas son menos productivas que las grandes tanto en España como en el resto de países, pero en España la pendiente de esta relación es más pronunciada: las pequeñas empresas en España son menos productivas que las homólogas europeas, mientras las grandes son equiparables o incluso más productivas.

La baja productividad del transporte terrestre se concentra principalmente en el transporte de mercancías por carretera pues las microempresas (las menos productivas) acumulan un porcentaje comparativamente alto de la producción total. Sin embargo el "Almacenamiento y Actividades Anexas" tiene una estructura más saneada en cuanto a productividad y dimensión de las empresas, aunque su producción es igualmente baja. Cabe concluir, por lo tanto, tres aspectos importantes con implicaciones de política:

- El camino del incremento de la dimensión de las empresas del transporte de mercancías por carretera para incrementar su competitividad es el adecuado y las políticas en este sentido de los últimos años no han producido todo el resultado esperable.

- El "Almacenamiento y Actividades Anexas" tiene una estructura saneada y su crecimiento debe provenir más del incremento de la demanda y de su expansión exterior.

- Es necesario "desacoplar", incluso mentalmente el crecimiento del sector del de los volúmenes de tráfico. El sector tiene un gran potencial de crecimiento (y de empleo). Pero este no se materializará en la forma de más movilidad.

Evidentemente, la ponencia analiza la anatomía del sector y no tanto su fisiología. La financiación, la incorporación de nuevas tecnologías para la comercialización y la gestión o la aportación de más valor a cada unidad transportada es la forma de recorrer el camino. 